\title{
POROCARCINOMA: UM RELATO DE CASO
}

\author{
POROCARCINOMA: ACASE REPORT
}

\author{
Helena Arantes Fiorilo Pelegrine ${ }^{1}$, Karina Demoner de Abreu Sarmenghi ${ }^{2}$, Priscila Andrade Tavares Dutra ${ }^{3}$, Thayla Baptista \\ Campostrini ${ }^{4}$, Paulo Bitterncourt de Miranda ${ }^{5}$, Hannah Cade Guimarães ${ }^{6}$ \\ ${ }^{1}$ Residente em Dermatologia do Hospital Santa Casa de Misericórdia de Vitória - ES. Graduada em Medicina pela \\ Universidade Federal do Espírito Santo - ES \\ ${ }^{2}$ Chefe do programa de residência médica em Dermatologia do Hospital Santa Casa de Misericórdia de Vitória - ES. Mestre \\ em Doenças infecciosas pela Universaidade Federal do Espírito Santo - ES. Dermatologista pela Sociedade Brasileira de \\ Dermatologia \\ ${ }^{3}$ Residente em Dermatologia do Hospital Santa Casa de Misericórdia de Vitória - ES. Graduada em medicina pela Faculdade \\ de Medicina de Campos - RJ \\ ${ }^{4}$ Residente em Dermatologia do Hospital Santa Casa de Misericórdia de Vitória - ES. Graduada em medicina pela Escola \\ Superior de Ciências da Santa Casa de Miséricórdia de Vitória - ES \\ ${ }^{5}$ Patologista atuante na área de Dermatopatologia do Hospital Santa Casa de Misericórdia de Vitória - ES. Patologista pela \\ Sociedade Brasileira de Patologia \\ ${ }^{6}$ Residente em Dermatologia do Hospital Santa Casa de Misericórdia de Vitória - ES. Graduada em medicina pela Escola \\ Superior de Ciências da Santa Casa de Miséricórdia de Vitória - ES \\ Intituição: Ambulatório de Dermatologia do Hospital Santa Casa de Misericórdia de Vitória ES - Brasil. Rua Dr. João dos \\ Santos Neves, 143, bairro Vila Rubim, Vitória - ES, Cep 29025-023
}

Autor responsável para troca de correspondência: Helena Arantes Fiorilo Pelegrine. Endereço: Rua Arnaldo Magalhães Filho, 201, apto 301, bairro Santa Lúcia, Vitória, ES, Cep 29056-125. Telefone: 27 981942993. Email: helenapelegrine@gmail.com

\section{RESUMO}

Introdução: O porocarcinoma écrino é uma rara neoplasia maligna de pele, originária das glândulas sudoríparas écrinas, que acomete principalmente idosos. A apresentação clínica é variada e os sítios mais comuns de ocorrência são cabeça e pescoço, seguido das extremidades inferiores.

Relato de caso: Paciente sexo masculino, com lesão nodular de $7 \mathrm{~cm}$ na região plantar direita, de longa evolução e que na investigação inicial apresentava metástase para linfonodos. Foi realizada radioterapia do sítio primário e da região linfonodal acometida. A cirurgia com intenção curativa não foi realizada, devido às metástases já presentes ao diagnóstico.

Discussão: O tumor não tem a etiologia bem definida, mas pode surgir como neoplasia primária ou como transformação maligna do poroma écrino. A biópsia é o principal método diagnóstico e os exames para estadiamento se impõem necessários, visto que até um terço desses tumores são met-astáticos. Acirurgia com amplas margens é o tratamento de eleição e terapias adjuvantes são reservadas para casos recorrentes e com metástases.

Conclusão: O diagnóstico precoce do tumor é necessário para evitar que a terapêutica ocorra em fases tardias e sem propostas curativas.

Palavras-chave: glândulas écrinas; Neoplasias das glândulas écrinas; Carcinoma

\section{ABSTRACT}

Introduction: Eccrine porocarcinoma is a rare skin neoplasm, origin of the eccrine sweat glands, which affects mainly elderly people. The clinical presentation is varied and the most common sites of occurrence are head and neck, followed by the lower extremities.

Case report: Male patient, with $7 \mathrm{~cm}$ nodular lesion in the right plantar region, of long evolution and that in the initial investigation presented lymph node metastasis. Radiotherapy was performed at the primary site and in the affected lymph node region. The surgery with curative intention was not performed, due to the metastases already present at the diagnosis.

Discussion: The tumor does not have the welldefined etiology, but may arise as primary neoplasm or as malignant transformation of the eccrine poroma. The biopsy is the main diagnostic method and the tests for staging are necessary, whereas one-third of these tumors are metastatic. Wide-margin surgery is the treatment of choice and adjuvant therapies are reserved for recurrent and metastatic cases.

Conclusion: The early diagnosis of the tumor is necessary to avoid that the therapy takes place in late phases and without curative proposals.

Keywords: Eccrine glands; Sweat gland neoplasms; Carcinoma 


\section{INTRODUÇÃO}

O porocarcinoma écrino, também chamado de poroma écrino maligno, tem origem do acrossiríngeo das glândulas sudoríporas. ${ }^{1}$ É um tumor raro, sendo que até o ano de 2017 foram relatados menos de 500 casos da neoplasia no mundo. ${ }^{2}$

Ocorre principalmente em idosos, sendo a idade média relatada de 67 anos. A frequência de ocorrência entre os gêneros é similar. ${ }^{2}$ Várias formas clínicas foram descritas, como pápulas, placas e lesões verrucosas, ${ }^{7}$ mas as principais formas de apresentação são os nódulos e massas. ${ }^{2} \mathrm{O}$ aspecto geralmente é eritematoso, podendo apresentar crostas e ulcerações ${ }^{1}$ e o tamanho médio é de $2,4 \mathrm{~cm}$.

Muitos pacientes são assintomáticos, mas dor, sangramento e prurido podem estar presentes. ${ }^{1,8}$ Os principais locais de acometimento são cabeça e pescoço $(39,9 \%)$, seguido pelas extremidades inferiores $(33,9 \%){ }^{2}$

O presente relato tem o objetivo de apresentar um tumor raro, de evolução arrastada, com implantes secundários ao diagnóstico, o que restringiu as possibilidades terapêuticas com finalidade curativa. Ressalta-se assim a importância dos dermatologistas e oncologistas no diagnóstico e seguimento adequado desses pacientes.

\section{RELATO DE CASO}

Paciente do sexo masculino, 67 anos, previamente hígido, relatava que há 30 anos, após trauma local, surgiu lesão de $3 \mathrm{~cm}$ na região plantar direita. Negava dor ou outros sintomas. A lesão permaneceu estável por longo período, apresentando crescimento significativo nos últimos 8 anos, dificultando a deambulação.

Ao exame físico inicial notava-se massa tumoral endurecida de aproximadamente $7 \mathrm{~cm}$, normocrômica, indolor à palpação, sem drenar secreção e com pequena exulceração. (FIGURAS 1 e 2 ). Observou-se linfadenomegalia inguinal direita de $2 \mathrm{~cm}$, indolor, endurecida e imóvel.

Foi realizada ressonância nuclear magnética do pé direito, evidenciando formação expansiva de part-es moles, medindo em seu maior eixo $6,9 \mathrm{~cm}$. A biópsia foi indicada, entretanto devido o tamanho e a localização não foi possível à excisão completa do tumor (FIGURA 3).

O estudo histopatológico, corado com hematoxilina-eosina, evidenciou neoplasia epitelial conectada a epiderme, exulcerada, constituída por blocos celulares de contornos infiltrativos, ocupando
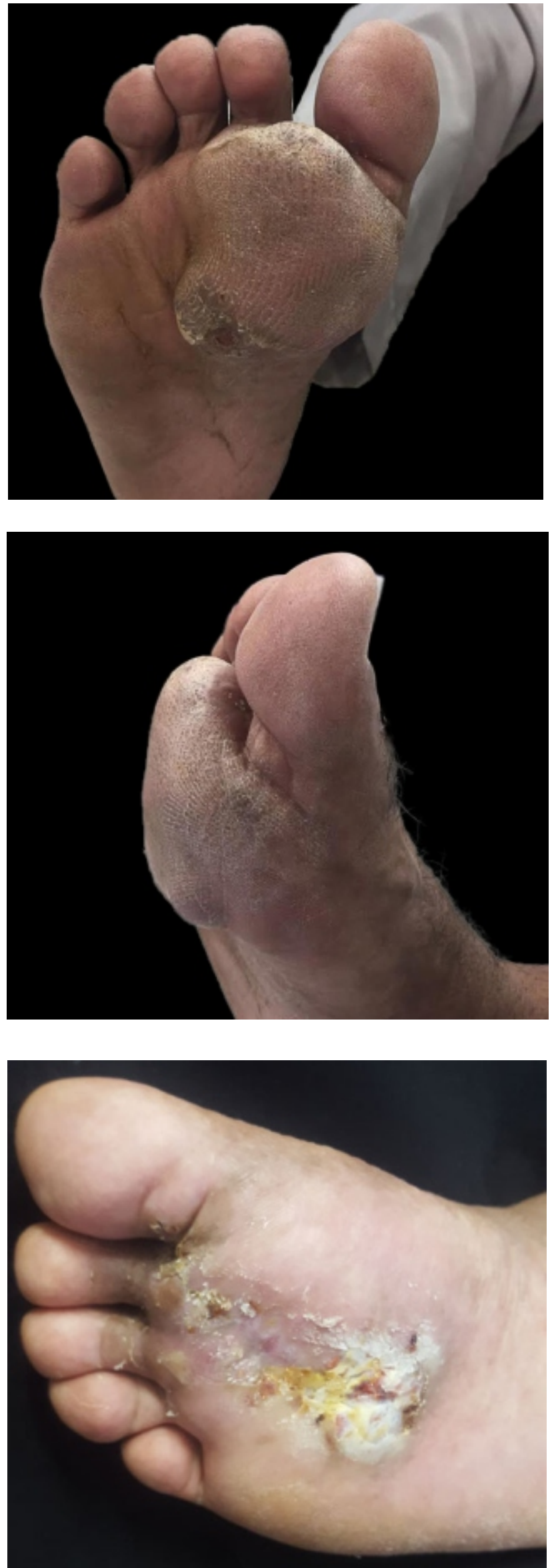
toda espessura da derme e atingindo o tecido adiposo subcutâneo. Notava-se lúmens de ductos e glândulas com material proteináceo secretório, no interior dos blocos neoplásicos. As células são epitelióides com citoplasma amplo a intermediário (aspecto poróide), por vezes pálidos, e os núcleos demonstram atipias. Visto também figuras de mitoses, focos de necrose, desmoplasia estromal leve, invasão perineural e ausência de evidência de invasão angiolinfática. (FIGURAS 4, 5 e 6). Os achados são compatíveis com o diagnóstico de porocarcinoma.

O paciente foi encaminhado para oncologia clínica para seguimento e estadiamento. A tomografia de tórax mostrava imagem nodular sugestiva de implante secundário. O linfonodo visualizado clinicamente foi submetido à punção aspirativa por agulha fina com resultado positivo para malignidade, optando-se pela linfadenectomia inguinal direita. Devido à presença de metástases já ao diagnóstico, não foi realizada a ampliação cirúrgica do sítio primário e foi indicada radioterapia inguinal e do pé direito. Paciente segue em acompanhamento e sem sinais de implantes em outros sítios.

\section{DISCUSSÃO}

A primeira descrição do porocarcinoma ocorreu em 1963, por Pinkus e Mehregan. A etiologia ainda não está bem definida, mas pode surgir como um tumor primário ou representar uma transformação maligna do poroma écrino. ${ }^{1-6}$ Quando tem origem primária, alguns fatores predisponentes podem estar associados, como radiação solar, imunossupressão e exposição a agentes químicos. ${ }^{2}$ Há relatos de tumores que surgiram após trauma local ${ }^{1}$ assim como ocorreu no presente caso.

Geralmente o tempo de duração até o diagnóstico é de aproximadamente 5 anos. ${ }^{2}$ Essa característica de curso arrastado entre o surgimento do tumor e o diagnóstico de malignidade reforça a hipótese de que muitos porocarcinomas surgem a partir de um tumor benigno, o poroma écrino. ${ }^{4}$

Entre os diagnósticos diferenciais estão o carcinoma basocelular, poroma écrino, carcinoma de células escamosas, queratose seborreica, granuloma piogênico e melanoma amelanótico. ${ }^{7-9}$

O diagnóstico é feito através da biópsia e estudo histopatológico, onde são detectadas alterações similares às descritas no atual relato. A Imunohistoquímica é útil quando se tem dúvida diagnóstica. Neste exame é vista coloração positiva para o antígeno carcinoembrionário (CEA) e para antígenos de membrana epitelial (EMA). ${ }^{9}$
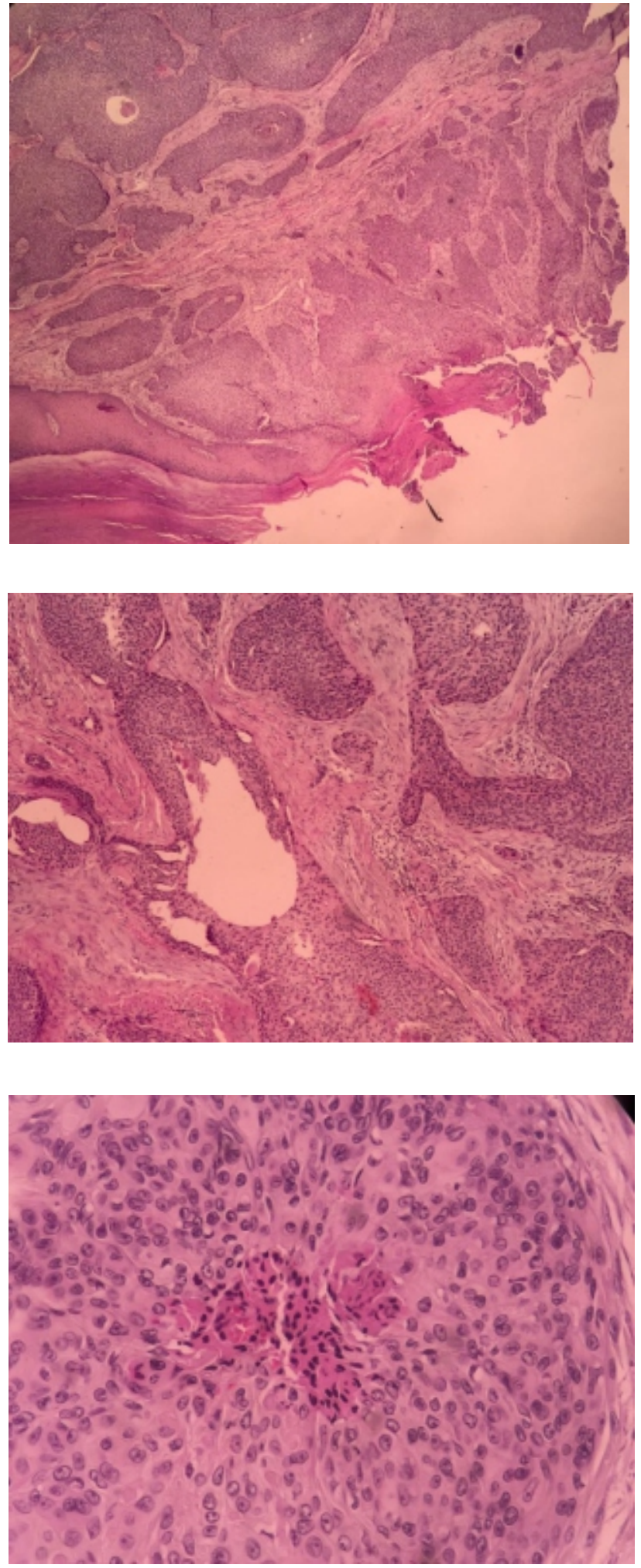

Considerando que muitos desses tumores surgem de uma transformação maligna do poroma écrino é importante ressaltar que a amostra da biópsia deve ser representativa, pois as células anaplásicas 
podem estar entre as células de aparência benigna do poroma, prejudicando o diagnóstico. ${ }^{1}$

O potencial de agressividade é considerável, com relato de $20 \%$ de recorrências locais e até $31 \%$ de metástases, sendo os nódulos linfáticos o sítio mais comum, seguido dos pulmões. ${ }^{2}$ No caso apresentado, o paciente já apresentava evidência de acometimento linfonodal e provavelmente pulmonar ao diagnóstico.

O tratamento de escolha é a ressecção cirúrgica total do tumor, com amplas margens, podendo alcançar cura em até $80 \%$ dos casos. ${ }^{3-5}$ Ainda não há protocolos bem estabelecidos para as terapias adjuvantes, sendo a quimioterapia e radioterapia reservadas para os casos metastáticos e recorrentes. ${ }^{2}$ Foi proposto um novo protocolo utilizando interferon alfa e isotretinoína para tumor metastático,${ }^{8,9}$ entretanto mais estudos são necessários. A linfadenectomia terapêutica é indicada no caso de presença de linfadenopatia, e a biópsia do linfonodo sentinela para estadiamento permanece discutível, ${ }^{2}$ podendo ser reservado para casos de agressividade histológica. ${ }^{3}$
No nosso relato, a radioterapia associado à linfadenectomia inguinal foi o tratamento escolhido, visto que o tumor já apresentava invasão linfonodal e provável metástase pulmonar, associado ao fato que a ampliação das margens cirúrgicas necessitaria de amputação do pé em paciente com doença agressiva.

Algumas referências citam uma sobrevida média de 5 a 24 meses ${ }^{4}$ e é descrita uma taxa de mortalidade de $67 \%$ em 5 anos em pacientes com metástases para linfonodos. ${ }^{1,3}$

\section{CONCLUSÃO}

Conclui-se que o porocarcinoma tem comportamento agressivo, sendo importante o seu diagnóstico precoce. Assim é imprescindível seu melhor conhecimento para evitar o início da terapêutica em fases avançadas e sem propostas curativas, como ocorreu no caso apresentado.

\section{REFERÊNCIAS}

1. Mulinari-Brenner JA, Mukai MM, Bastos CAS, Amaral Filho EA, Santamaria JR, Fillus Neto J. Porocarcinoma écrino: relato de quatro casos e revisão da literatura. An Bras Dermatol. 2009;84(5):519-23

2. Salih AM, Kakamad FH, Baba HO, Salih RQ, Hawbash MR, Mohammed SH, et al. Porocarcinoma; presentation and management, a meta-analysis of 453 cases. Annals of Medicine and Surgery 20 (2017) 74-79

3. Marone U, Caracò C, Anniciello AM, Monta G, Chiofalo MG, Cecilia ML, Mozzillo N. Metastatic eccrine porocarcinoma: report of a case and review of the literature. World Journal of Surgical Oncology 2011, 9:32

4. Mercadillo-Pérez P, Morales-Trujillo ML, Moreno-López LM, Peniche-Castellanos A. Porocarcinoma ecrino. Reporte de un caso. Rev Med Hosp Gen Mex 2010; 73 (1): 39-42

5. Acosta-Arencibia A, Abrante-Expósito B, Ramos-Gordillo M. Multiple recurrent eccrine porocarcinoma with inguinal metastasis. A case report. Cirugía y Cirujanos. 2016;84(1):73-76

6. Melgandi W, Benson R, Hakin A, Bhasker S. Porocarcinoma scalp with high risk features treated with surgery and adjuvant radiotherapy: A case report and review of literature. Journal of the Egyptian National Cancer Institute (2016) 28, 195-198

7. Cursino FDU, Lima EA, Rodrigues SCC, Teixeira L, Lima MA, Takano D. Porocarcinoma - Relato de caso. An Bras Dermatol. 2011;86(6):1201-4

8. MS Lloyd, N El-Muttardi, A Robson. Eccrine porocarcinoma: A case report and review of the literature. Can J Plast Surg 2003;11(3):153-156.

9. Luz MA, Ogata DC, Montenegro MFG, Biasi LJ, Ribeiro LC. Eccrine Porocarcinoma (Malignant eccrine poroma):A series of eight challenging cases. Clinics 2010;65(7):739-42 